\title{
Quality in anesthesiology - a demand more than current
}

Volume 3 Issue 4 - 2015

\section{Introduction}

Anesthesiology was born in the mid-19th century because the medicine needed to provide patients the relief of acute pain. This speciality has, in its wake, the brand with the patient care. Although the concern with the quality of anesthesia is present from the beginning of its history, the systematic quality control methods had great momentum only at the end of the last century. ${ }^{1}$ The quality, extracted and industry concept applied to medicine, has evolved constantly in recent years. Consists of a process that begins with the identification of problems and their quantification, which use indicators of structure, procedures and results. ${ }^{2}$ In the area of health, quality management models emerged in developed countries with the aim of reducing costs. With this approach, failure happened then to focus on the results of the assistance processes to ensure patient satisfaction. ${ }^{3}$ Avedis Donabedian sentences that quality in health can be defined as the ability of professionals, hinged system of health services and the society set up a harmonic set able to offer credible assistance, technically developed by trained professionals and paid , being all this set financially viable, economically sustainable and

\author{
Alberto Tirelli \\ Anesthesiologist São Lucas Hospital/PUCRS, Brazil
}

\begin{abstract}
Correspondence: Alberto Tirellli,Anesthesiologist at the São Lucas Hospital, Member of the Board of Anesthesiology from Hospital Moinhos de Vento, MBA in Health Audit, President of the Society of Anesthesiology of Rio Grande do Sul (SARGS), Eduardo Prado Ave, 2050/25, Porto Alegre-Rio Grande do SulBrazil, Zip Code: $91.751-000$, Tel 55 51 998I-2242,

Email atirellil990@hotmail.com
\end{abstract}

Received: November 21, 2015 | Published: November 25, 2015

a democratic choice of citizenship. ${ }^{4}$ This article aimed to introduce quality concepts and indicators of quality control in Anesthesiology, through a bibliographical review of the contemporary theme, with emphasis on information published between the years 2005 and 2010.

Table I Quality components (according to Avedis Donabedian)

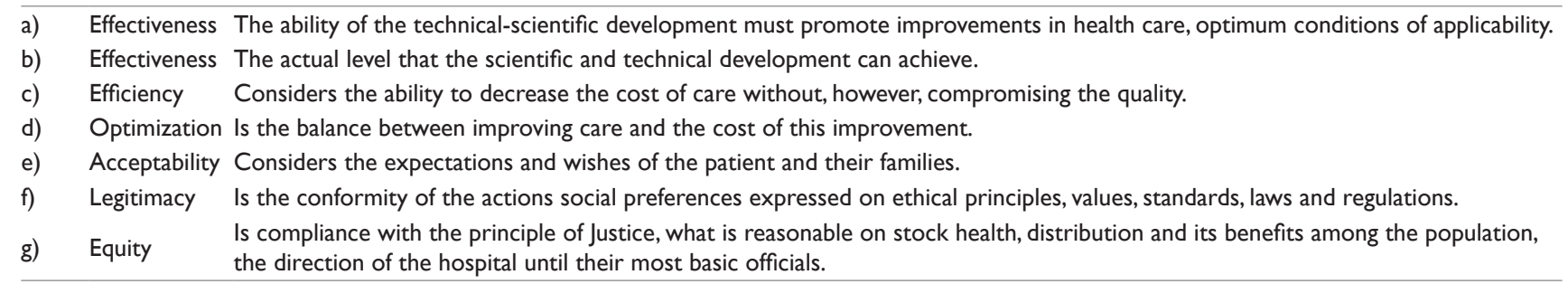

The literature review for this article was conducted through research done in articles, magazines, publications and studies until 2010, in addition to research performed on electronic databases: Medline/PubMed ${ }^{5}$ and Virtual Library of Brazilian society of Anesthesiology (SBA- www.sba.com.br/educa/bv.asp). ${ }^{6}$ The selection criteria of the research material for the creation of the study followed four steps: the first was the research of the keywords: quality, or quality, and Anesthesiology, or anesthesiology; in the second, set limits on the period of publication of the articles, set between 2005 and 2010, taking into account the contemporaneousness of scientific findings; in the next step, other limits were defined as articles published in English, Portuguese and Spanish languages; in the last step of the survey, selected articles as full text but only with the summary available.The research methodology is recommended by The Cochrane Collaboration Library. ${ }^{7}$ It is important to note that the keyword search was conducted in several medical databases, as Scientific Electronic Library online (Scielo) and Latin American literature and Caribbean Health Sciences (Lilacs), and literature in these databases or not filled the criteria proposed by the study for the inclusion of the work or was not available. Quality concepts Avedis Donabedian believes that quality in health can be defined as the ability of professional services, articulated system of health and society in configure a harmonic set able to offer credible assistance, technically well developed, by trained professionals and fairly paid, that users can use it whenever and to the extent of their needs, being all this set financially viable, economically sustainable and a democratic choice of citizenship (Table 1$)^{4}$ Also understand quality as a dynamic process continuous and comprehensive, permanent identification activity of faults in the routines and procedures, which must be periodically reviewed, updated and disseminated, with the participation from high.

In more modern concepts, the term quality is a continuous phenomenon of improvement, establishing, progressively, patterns, a result of studies of historical series in the same organization or in similar organizations (benchmarking), in search of defect zero. ${ }^{3-}$ ${ }^{8}$ The patient-oriented quality shelter, in addition to improvements in structure (anesthetics, equipment, human capital etc.) and results (efficiency in the desired outcome), improvements of processes in which the practice occurs (streams, routines, etc.). As ongoing evaluation of technical quality of health professionals in our country is a controversial topic, the Brazilian Medical Association establishes the Professional Upgrade Certificate for bondholders of specialist, considering the rapid contribution and the incorporation of new knowledge in medical practice. ${ }^{4}$ Accreditation and certification Accreditation is a word originating in the English language which is used by Brazilian Accreditation Manual and the Manual of 
Organizations Providing Hospital Services. For the World Health Organization (WHO), as of 1989, the accreditation has become strategic element for the development of quality in Latin America. Is a system of evaluation and certification of quality of health services volunteer, periodic and reserved. Have an educational character, focused on continuous improvement, and has advantages, such as security for patients and health professionals, quality of service and team building, being useful management tool, with specific criteria and objectives adapted to Brazilian reality. ${ }^{9}$ The greater interested by the accreditation processes are health organizations, health professionals, administrators and leaders the Government and the citizens. ${ }^{9}$ The accreditation or certification systems of quality are methodologies that allow you to audit a company or service, by a third, which so measurable, it can be said that it is able to provide quality assistance. ${ }^{3-8}$ In 1995, in Rio Grande do Sul, the Institute of Hospital Management and Health Sciences, in association with the Department of Health and the Environment and Sebrae/RS , has developed a research project with the intention to determine hospital quality standards. In 1999, the National Accreditation Organization (NAO), which is a private, nonprofit organization and collective interest, that has as main objective the national deployment of a permanent process of improving the quality of health care, being responsible for hospital quality assessment. ${ }^{3-8}$ The institutions are law firms certification accredited by the ONA to have the responsibility to carry out the evaluation and certification of quality of health services at the national level. The certifiers, such as ISO and the Joint Commission International (JCI), have different methodologies of evaluation..$^{3-8}$ Indicators in Anesthesia Set quality, we must introduce this monitoring instruments, called indicators, which can be understood as a quantitative representations of results, being in powerful management tool for monitoring, measurement and quality assessment, as the Donabedian model, are classified as indicators of structure, process and results. ${ }^{10}$ Indicators are quantifiable forms of representation of processes and products that aim to control and improve the quality and performance of them. However, to be useful, the indicators must be, in addition to measurable, achievable. That is, the indicator is the variable that describes the element from the quantitative point of view situation. ${ }^{3}$ Indicators can assist the health institutions in the quest for quality, control of processes, in structuring a database reliable and useful for strategic planning and decision making. ${ }^{11}$ The numb--wow-Physiology no exception, so that the anesthesia services must establish its quality indicators to better monitor the structure, the process and the result. ${ }^{4}$ Examples of quality indicators to be monitored in a quality control program for a service of anesthesia are: global indicators (total number of anesthesia, number of anesthetized patients, number of patients by specialty, number of locorregionais anesthesia, number of interventions cancelled, emergency assistance number, number of anesthesia for surgery, length of stay in the surgical room , percentage by gender [female/ male], middle ages and age extremes, anesthesia in children under one year, anesthesia in the elderly above 75 years, ASA classification and adverse events [nausea, vomiting, postoperative pain]); preanesthetic assessment (number of preoperative consultations/ patients anesthetized, number of queries in the hospital bed, number of queries for the benefit of another institution); ambulatory anesthesia (number of ambulatory anesthesia, number of procedures canceled, number of hospitalizations not provided); Obstetric Anesthesia (number of normal births, number of obstetric anesthesia, cesarean section number, percentage of Cesarean sections under general anesthesia, spinal anesthesia and epidural, epidurals for obstetric analgesia number, number of queries in the third quarter, number of obstetric anesthesia without consulting the distance); recovery (number of patients admitted to the post-anesthetic Recovery room [PACU], number of patients admitted directly in intensive care and length of stay in the RECOVERY ROOM). ${ }^{3}$ In a systematic review published in Anesthesiology, the authors identified 108 clinical indicators developed to measure quality and safety in anesthesia. About half of them could be considerate of specific to the specialty. Most eraindicadores (57\%) or processes (42\%). Only $1 \%$ was related to structures. ${ }^{12}$ Condi important to the construction of indicadores to obtain reliable data and information be understood comorepresentaes quantitativasde resultadosoriundos Indicators of well-executed anotaesprecisas reports of the professionals involved. ${ }^{10}$

\section{Conclusion}

Health services are in a difficult phase, and the implementation of quality programs and the consequent certification/accreditation of extreme importance, as will provide the reduction of costs and rates of morbidity and mortality, leading to the satisfaction of a larger portion of the population. The total quality is something able to cause theoretical discussions and a challenge to its practical application. For this reason, you should continue to be studied to develop management models adapted to the peculiarities of the institutions. The participation of Anesthesiology in accreditation programs contributes to the personal and institutional improvement and the improvement of the service provided by specialists. ${ }^{3}$ Thus, it becomes increasingly important to engage in institutional programs anesthesiologists and their participation in activities and events promoted by the quality management. ${ }^{4}$ Declare if any financial interest or any conflict of interest exists.

\section{Conflicts of Interest}

The authors do not have any Conflict of interests.

\section{Acknowledgments}

None.

\section{Funding}

None.

\section{References}

1. Burmester H. Amaral JLG-quality management in Anesthesiology. In: Manica J, â€" Anesthesiology, principles and techniques. 3rd edn, Porto Alegre, Artemed, Germany. 2004;1001-1008.

2. Borel J. Sivanto M-Gestion de calidad in anestesiolog. Rev Argent Anestesiol. 2008;1:71-72.

3. Slullitel. quality management in Anesthesiology. Hospital practice. 2008;58:93-96.

4. Diego LA. Anesthesiology and quality care. Anesthesia review. 2009;4:18-25.

5. National Library of Medicine.

6. The Cochrane Collaboration Library.

7. Feldman LB. Gatto MAF ICKO Wedge-the evolution of hospital quality: accreditation standards. ACTA Paulista of nursing. 2005;18:213-219.

8. Virtual Library of Brazilian society of Anesthesiology.

9. ONA-national accreditation Organization.

10. Duarte IG, Ferreira DP. use of indicators in the management of a surgical Center. Rev adm health. 2006;63-70.

11. Treviso P, Brand FH, Saitovitch D. Construction of indicators in health services. Rev adm health. 2009;11:182-186.

12. Haller G, Stoelwinder J, Myles. Quality and safety indicators in anesthesia: a systematic review. Anesthesiology. 2009;110: 1158-1175. 